\title{
I. Kirche und Besatzungsmacht
}

Erste Erfahrungen mit deutschen Kirchenführern gewannen die amerikanischen Besatzungstruppen im Oktober 1944 nach der Besetzung Aachens. Der katholische Bischof von Aachen, Johannes van der Velden, erklärte sich sofort zur Zusammenarbeit bereit und gab zu verstehen, daß er den Klerus anweisen werde, ,,den US-Truppen Auskünfte über die einheimische Bevölkerung zu geben und sie vor Nazis zu warnen. Er wünscht jedoch", heißt es im Gesprächsprotokoll weiter, ,,mit allem Nachdruck, daß jede derartige Zusammenarbeit völlig geheimgehalten werden müsse, da weder er noch irgendeiner seiner Priester Lust hätten, vor ihren Landsleuten als ,Denunzianten ' dazustehen. “ Nicht minder nachdrücklich sprach sich van der Velden, als er nach seinen Vorstellungen hinsichtlich der Zukunft Deutschlands befragt wurde, für die konstitutionelle Monarchie oder für eine Republik mit starkem Präsidenten nach amerikanischem Vorbild aus, da "der Staatstyp der Weimarer Republik der deutschen Mentalität nicht nahezubringen" sei. Das neue Deutschland solle ,,von allen konservativen Kreisen, katholischen und anderen, gestaltet werden, um es gegen die Gefahr eines Wiedererstehens des Nazismus oder einer kommunistischen Revolution zu schützen". Hinsichtlich der Entnazifizierung erklärte der Bischof, viele Beamte seien aus Charakterschwäche der NSDAP beigetreten, könnten aber gleichwohl ,,nach sorgfältiger Prüfung mit großem Nutzen von den Besatzungsbehörden beschäftigt werden" "1.

Die positiven Reaktionen, die der erste Kontakt bei den Amerikanern hervorrief, wurden nachhaltig getrübt, als ein Team der Psychological Warfare Division die Personalpolitik des neuen Oberbürgermeisters Franz Oppenhoff untersuchte, den van der Velden vorgeschlagen hatte. Oppenhoff hatte, ganz im Sinne einer Neugestaltung Deutschlands auf konservativer Grundlage, die Stadtregierung aus konservativen Fachleuten und jungen Industriellen gebildet und dabei bewußt auf die Einbeziehung von SPD- oder KPDVertretern verzichtet. Der Führungsspitze gehörte zwar nur ein NSDAP-Mitglied an, doch waren von 72 Schlüsselpositionen in der Verwaltung nicht weniger als 22 mit Parteigenossen besetzt. Dieser Befund löste bei dem linksorientierten Untersuchungsteam unter der Leitung Saul K. Padovers heftige Empörung aus: ,,In short, under the nose of MG, the Oppenhoff administration was setting up the framework of an authoritarian, hierarchical, bureaucratic, corporate fascism - a type of Ständestaat that even the Nazis had rejected." 2 Der Bericht warf der örtlichen Militärregierung politische Naivität und Diskriminierung der Antifaschisten vor und löste, da er auch der amerikanischen Presse zugespielt wurde, scharfe Reaktionen aus. Noch kurz vor der Ubergabe Aachens an die Briten kam es zu einer Entlassungswelle in der Stadtverwaltung, der sich die Degradierung des Aachener MG-Detachements anschloß. Oppenhoff, der auf seinem Posten belassen worden war, wurde am 25. März 1945 von zwei SS-Männern als Kollaborateur ermordet.

${ }^{1}$ Protokoll vom 20.10.1944, in: Volk, Ausblick, S. $205 \mathrm{ff}$.

2 Zit. nach Saul K. Padover, Experiment in Germany. The Story of an American Intelligence Officer, New York 1946, S. 224. Vgl. Niethammer, Besatzungsmacht, S. 172 ff. 
Das Beispiel Aachen ist in vieler Hinsicht für die ersten Besatzungsmonate typisch. Es zeigt den Einfluß der Kirchen auf die Neubesetzung von Stadt- und Gemeindeverwaltungen, ihre Förderung konservativer Kreise, die auch eine Protektion kirchentreuer Nationalsozialisten einschloß, wie die Bedeutung der amerikanischen Presse, die echte oder vermeintliche Entnazifizierungsskandale begierig aufgriff und die Militärregierung auBerordentlich unter Druck setzen konnte. Gleich in der ersten Stadt unter amerikanischer Besatzung zeigte sich auch der Konflikt zwischen der politischen Säuberung, deren Kriterien nur vage definiert waren, und der Erhaltung administrativer Effizienz.

Mit dem Vordringen der amerikanischen Truppen wurde das Problem, nach welchen politischen Kriterien Neubesetzungen erfolgen sollten, immer dringlicher, zumal amerikanische Linksintellektuelle und Emigranten in höheren Militärstäben die Zusammenarbeit des zumeist konservativ eingestellten Offizierskorps der Kampftruppen mit deutschen Konservativen auf lokaler Ebene mit großem Mißtrauen verfolgten. Im März 1945 wandte sich Brigadegeneral Frank McSherry hilfesuchend an Robert Murphy, den politischen Berater des State Department für die amerikanische Militärregierung, und forderte mit Verweis auf die Ereignisse in Aachen konkrete Richtlinien: ,, There is crying need for guidance. "3 Anfang Mai übermittelte Murphy dem amerikanischen Außenminister einen Richtlinienentwurf, der neben den geltenden Entnazifizierungsbestimmungen auch positive Auswahlkriterien enthielt: Bevorzugt eingestellt werden sollten Funktionäre aus den demokratischen Organisationen der Weimarer Zeit. Hierzu rechnete Murphy die Gewerkschaften, SPD und DDP sowie viele Mitglieder des Zentrums. Auch unter den Mitgliedern der DVP und DNVP könnten sich Antinationalsozialisten befinden, ,, but they were so generally imbued with German nationalism, militarism, and conservative traditionalism as to make their members unsuitable for all purposes. In many cases members of these rightist parties are only less dangerous than the Nazis themselves. "Weiterhin empfahl Murphy bei Neubesetzungen alle demokratischen Gruppierungen angemessen zu berücksichtigen: , Appointments should especially avoid giving too much prominence to elements from extreme left or extreme right of the anti-Nazi-section of the population." In vornehmlich katholischen Gebieten sei es angebracht, mehr ehemalige Zentrumsmitglieder zu ernennen, während in protestantischen Industriegebieten Sozialdemokraten und Gewerkschafter bevorzugt werden sollten. Der Rat der Ortspfarrer sei vielfach nützlich, doch müsse die örtliche Militärregierung jeden Kandidaten sorgfältig überprüfen, da die Kirchen keineswegs gänzlich anti-nationalsozialistisch eingestellt gewesen seien ${ }^{4}$.

Ausführlicher führte Murphy seine politische Beurteilung der deutschen Kirchen in dem Antwortschreiben an McSherry aus: Die evangelische Kirche besitze zwar einige herausragende Führer, die dem Nationalsozialismus Widerstand geleistet hätten, habe sich jedoch in weiten Teilen, besonders in den späteren Jahren der NS-Herrschaft, dem Druck der NSDAP unterworfen. Âhnliche Vorbehalte äußerte Murphy, selbst praktizierender Katholik ${ }^{5}$, auch hinsichtlich der katholischen Kirche. Sie habe zwar im ganzen eine geschlossenere Front gegen den Nazismus gebildet als die evangelische Kirche. Neben couragierten Anti-Nationalsozialisten wie den Bischöfen Preysing in Berlin, Galen

\footnotetext{
${ }^{3}$ NA, RG 84, 731/3, McSherry an Murphy vom 22.3.1945.

${ }^{4}$ NA, RG 84, 737/3, Draft Directive: Political Considerations for the Guidance of Military Government Officers in Making Appointments in Germany vom 7.5.1945.

${ }^{5}$ Spotts, Kirchen, S. 52.
} 
in Münster und Faulhaber in München habe es jedoch auch viele gegeben, die keine ähnlich kompromißlose Haltung eingenommen hätten und die deshalb nicht als vertrauenswürdige Antinazis gelten könnten. Die Erfahrung habe gezeigt, daß die von Pfarrern empfohlenen Personen zumeist konservativen Kreisen angehörten. ,, We would thus lay ourselves open to the charge of encouraging, rightist' and ,reactionary' political movements and policies at the expense of more, liberal' policies if we leaned too heavily or exclusively on church advice. We must constantly bear in mind the point [...] that while many of the rightist and conservative elements were anti-Nazi, they were at the same time strongly nationalistic and imbued with the German military tradition. Our aim is not only to destroy Nazism (negative) but to seek out and encourage elements which have been or may become truly democratic (positive). " 6

Mit der geforderten demokratischen Gesinnung war es jedoch häufig nicht zum Besten gestellt, wie spätere Ubberprüfungen der von den amerikanischen Kampftruppen ad hoc eingesetzten Amtsträger ergaben. Auch die kirchlichen Personalvorschläge entsprachen oft nicht den politischen Anforderungen eines demokratischen Neubeginns. Leo Schwering, ein führender katholischer Politiker aus dem Rheinland, urteilte am 13. April 1945 in seinem Tagebuch über die Personalpolitik der Pfarrer: ,,Sie empfehlen Personen, die politisch bedenklich sind, denen sie aber Vertrauen schenken, weil sie gute Katholiken sind [...]. So erlebt man, daß eine ganze Reihe von richtigen Nazis in wichtigen Stellungen sitzen, [...] gedeckt durch den schwarzen Rock und dessen Empfehlungen schmuggeln sie sich ein."

Nicht viel anders verhielten sich evangelische Würdenträger. Wie gering das Reservoir völlig unbelasteter Kandidaten mit einwandfreier demokratischer Vergangenheit war, zeigte sich, als Colonel Colberg, der Leiter der Rechtsabteilung der bayerischen Militärregierung, am 4. Juni den bayerischen Landesbischof Hans Meiser aufsuchte und ihn um die Benennung vertrauenswürdiger Personen zur Reorganisation des Justizwesen bat. Meiser erklärte unumwunden, daß er ,,nur dann eine Liste geeigneter Juristen vorlegen könne, wenn Parteigenossen nicht von vornherein ausgeschlossen sein sollen" ${ }^{\text {"8 }}$. Ahnlich verlief die Unterredung zwischen dem württembergischen Militärgouverneur Dawson und Landesbischof 'Theophil Wurm am 19. Juli, als es um die Benennung geeigneter Persönlichkeiten für die württembergische Landesregierung ging: ,, Der Herr Bischof erklärt zurückhaltend, es sei schwer, Leute vorzuschlagen, weil fähige Leute und die Mittelschicht (altersmäßig), sofern sie nicht Parteigenossen gewesen seien, kaum Gelegenheit gehabt hätten, sich zu entfalten und zu bewähren. " Besorgt über die ersten Entlassungsmaßnahmen der Militärregierung, stellte Wurm auch die Frage, ob es denn wahr sei, daß keine NSDAP-Mitglieder mehr als „,Bürgermeister, Landräte etc.“ amtieren dürften”.

Im Unterschied zur katholischen Kirche, die kaltgestellte Zentrumspolitiker oder ehemalige Funktionäre der christlichen Gewerkschaften für Spitzenpositionen benennen konnte, hatten die evangelischen Bischöfe große Schwierigkeiten, den ihnen zugestandenen politischen Einfluß zu nutzen, da sie nur in sehr geringem Umfang auf bewährte Per-

6 NA, RG 84, 731/3, Murphy an McSherry vom 4. 5.1945.

7 Zit. nach Spotts, Kirchen, S. 86.

8 LKAN, NL Meiser 212, Aktennotiz Meisers vom 4. 6. 1945. Vgl. Kap. IV/2.

${ }^{9}$ LKAS, NL Hartenstein, 52/3, Bericht über Empfang des Landesbischofs beim amerikanischen Militärgouverneur am 19.7.1945. 
sönlichkeiten aus dem demokratischen Spektrum der Weimarer Parteienlandschaft zurückgreifen konnten. Parteipolitisch hatte sich die überwiegende Mehrheit der evangelischen Pfarrerschaft und der sogenannten ,,gut kirchlichen Kreise" während der Weimarer Republik an den antidemokratischen Rechtsparteien, vornehmlich der DNVP, orientiert $^{10}$. Das Leitbild der mit wenigen Ausnahmen fest im konservativ-nationalen Lager beheimateten Meinungsführer in Theologie, kirchlicher Presse und kirchlichem Verbandswesen war und blieb die verklärte Welt des preußisch-protestantisch geprägten Wilhelminischen Kaiserreichs. Den Aufstieg der NSDAP hatten maßgeblich evangelische Wählerschichten getragen. Wie Wahlanalysen zeigen, wurde nach 1930 der Konfessionsfaktor ,,zur wichtigsten Einflußgröße des nationalsozialistischen Wählerverhaltens überhaupt" "11. Erst im März 1933 gelangen der NSDAP nennenswerte Einbrüche in katholische Gebiete, die bis dahin eine kaum zu überwindende Barriere dargestellt hatten.

Einen Einblick in den geistigen Horizont evangelischer Kirchenführer unmittelbar bei Kriegsende vermitteln die Aufzeichnungen hoher amerikanischer Offiziere über erste Begegnungen und Gespräche mit Vertretern der Bekennenden Kirche. Sie hinterließen bei den Kirchenoffizieren der Education and Religious Affairs Branch (ERA) einen recht zwiespältigen Eindruck. Starke Zweifel an der Demokratiebereitschaft des deutschen Protestantismus waren Marshall M. Knappen, dem Leiter der Religious Affairs-Abteilung der amerikanischen Militärregierung, bereits während der gemeinsamen anglo-amerikanischen Planungsphase 1944/45 gekommen ${ }^{12}$.

Genügend Anlaß für solche Vorbehalte boten die Lektüre der Zeitschrift der Bekennenden Kirche (Junge Kirche), die den Kampf des Nationalsozialismus gegen den gottlosen Kommunismus stets lobend hervorhob, und Niemöllers 1934 erschienene Autobiographie ,,Vom U-Boot zur Kanzel", die Sympathien für die NS-Bewegung deutlich erkennen ließ ${ }^{13}$. Niemöllers Vergangenheit als Marineoffizier und Freikorpsführer während der Ruhrkämpfe 1920 bestärkte Knappen noch zusätzlich in der Einschätzung, daß die Bekennende Kirche, die als Widerpart der Deutschen Christen gewissermaßen den Hoffnungsträger des deutschen Protestantismus darstellte, unter politischen Gesichtspunkten als ausgesprochen konservativ und illiberal zu beurteilen sei: ,, Not all the anti-

${ }^{10}$ Dahm, Pfarrer, S. 148, rechnet 70-80 Prozent der evangelischen Pfarrerschaft zum Typ des konservativ-national eingestellten Pfarrers mit ausgeprägt antidemokratischer Gesinnung. Vgl. auch Nowak, Kirche und Weimarer Republik; Jacke, Kirche zwischen Monarchie und Republik; Wright, Uber den Parteien; Scholder, Kirchen, Bd. 1.

${ }^{11}$ Falter, Wer verhalf der NSDAP zum Sieg?, S. 16. Vgl. Scholder, Kirchen, Bd. 1, S. $160 \mathrm{ff}$. Die Bedeutung des Konfessionsfaktors bleibt in abgeschwächter Form auch dann erhalten, wenn man für die protestantischen Gebiete Nord-, Mittel- und Ostdeutschlands, in denen die NSDAP ihre größten Erfolge erzielen konnte, die Negativfaktoren - höhere Arbeitslosigkeit, stärkere Agrarverschuldung und geringerer Urbanisierungsgrad - berücksichtigt. Vgl. Loren $\mathrm{K}$. Waldmann, Modells of Mass Movements - The Case of the Nazis, Diss. Chicago 1973, S. 79-99. Zahlreiche Beispiele für den protestantischen Zeitgeist 1933 finden sich bei van Norden, Kirche in der Krise, und Stoll, Zeitschriftenpresse.

12 Zur amerikanischen Kirchenpolitik vgl. Boyens, Kirchenpolitik; Scheerer, Kirchen, S. 12ff.; Vollnhals, Alliierte Kirchenpolitik; Vollnhals, Reichskonkordat. Zur Organisationsgeschichte der ERA-Branch vgl. Tent, ERA-Branch; Tent, Mission, S. $46 \mathrm{ff}$.; Einführung Josef Henkes in den ECR-Bestand (NA, RG 260, 5/291-3). Zur britischen Kirchenpolitik vgl. Besier, Selbstreinigung.

13 Vgl. Schmidt, Niemöller, S. $40 \mathrm{ff}$. 
Nazi elements in the German churches should be fairly called democratic and reasonable be expected to cooperate with a future democratic program." 14

Wenn es noch eines spektakulären Anlasses zur Bestätigung dieser Vorbehalte bedurfte, so lieferte ihn Niemöller in einem aufsehenerregenden Interview, das er kurz nach seiner Befreiung aus dem Konzentrationslager alliierten Reportern während eines Erholungsurlaubs in Neapel gab. Am 5. Juni teilte „Hitlers persönlicher Gefangener“ einer staunenden Weltöffentlichkeit in fließendem Englisch mit, daß er sich im September 1939 erfolglos aus dem Konzentrationslager Dachau zur Kriegsmarine gemeldet habe. Weiterhin erklärte Niemöller, er habe sich dem Totalitätsanspruch der NS-Ideologie aus rein religiösen Gründen widersetzt und sich als guter Lutheraner verpflichtet gefühlt, für sein deutsches Vaterland zu kämpfen: ,My soul belonged to God. But I must render into Caesar things, that are Caesar's, which is my whole physical life." Damit nicht genug, erklärte Niemöller, daß für das deutsche Volk eine Demokratie nach angelsächsischem $\mathrm{Mu}$ ster nicht in Frage komme: ,,The Germans like to be governed. They like to live under some authority and not to be mingled into politics and such things. [...] That sort of democracy which would be useful for Germany must be found. "15 Schließlich gab Niemöller noch bekannt, daß er nach England und in die USA reisen wolle, um Geld und Lebensmittel für das hungernde deutsche Volk zu sammeln. Niemöllers Auftritt hätte wohl kaum katastrophaler ausfallen können, war er doch im westlichen Ausland zum demokratisch gesinnten Märtyrer des christlichen Gewissens gegen die Nazi-Barbarei verklärt worden. Da der Widerstand der Bekennenden Kirche gegen die Deutschen Christen im Ausland zumeist als politisch motivierter Widerstand gegen das NS-Regime interpretiert worden war, mußte das Interview heftige Irritationen auslösen. Wie mußte es um das deutsche Volk bestellt sein, wenn selbst verfolgte Pfarrer bereit waren, freiwillig für Hitler zu kämpfen, und nach der militärischen Niederringung des NS-Regimes erklärten, eine demokratische Staatsform sei für das deutsche Volk nicht geeignet? Bestätigte sich damit nicht die These des Vansittarismus, alle Deutschen seien verkappte Nazis? In der amerikanischen Militärregierung war man derart schockiert, daß Niemöller in einer Uberreaktion sofort als unverbesserlicher Nationalist festgesetzt wurde.

Am 18. Juni führte Knappen ein langes Gespräch mit Niemöller, an dem auch zwei Mitarbeiter des amerikanischen Geheimdienstes OSS teilnahmen. Die Begründung, die Niemöller nun für seine Frontmeldung gab, charakterisiert anschaulich das Dilemma, in das die Bekennende Kirche 1939, wie vor ihr 1914 die Arbeiterbewegung, geraten war: „Up to the outbreak of war, Niemöller stated, he was opposed to it, but once it had begun he could see no alternative than to take up the fight of one's nation. It was particulary easy to adopt this position, he said, because the ,Polish terror' just previous to outbreak had been real. He claimed to know many German Protestant clergymen who had been ,slaughtered" in Poland. Accordingly he had volunteered for active service. "Erst im KZ Dachau (ab 1941) habe er begriffen, daß Hitler nicht nur ein Fanatiker, sondern ein Verbrecher sei; von da an habe er für die Niederlage der Nazis gebetet. Auf die Frage nach

${ }^{14}$ NA, RG 260, 5/340-2/10, Knappen, Historical Report. Education and Religious Affairs. Winter 1941 - Spring 1946, S.10. Vgl. Knappen, Peace, S. 49 f.

15 Zit. nach Daily Sketch, Niemoeller offered to fight us, und New York Herald Tribune, Niemoeller sees Germany unfit for Democracy on U.S. Lines, vom 6.6.1945. Vgl. Bentley, Niemöller, S. $192 \mathrm{ff}$. 
seiner Einstellung zum sogenannten Röhm-Putsch 1934, als Hitler hohe SA-Führer und konservative Gegner ermorden ließ, antwortete Niemöller, er habe damals fest an eine Verschwörung geglaubt und deshalb das Handeln der Nazis teilweise für berechtigt gehalten ${ }^{16}$. Zur gegenwärtigen politischen Lage führte Niemöller aus, Deutschland werde wohl bald vollständig unter russischen Einfluß geraten, teils weil die amerikanischen Truppen in Europa viel zu schwach seien, teils weil der „Bolschewismus“ neben dem Christentum die einzige geistige Kraft in Deutschland darstelle. Auch sei der Anschluß Deutschlands an die Sowjetunion innerhalb der nächsten sechs Monate eine denkbare Entwicklung, die er nicht begrüße, aber auch nicht von vornherein ablehnen wolle. Weiterhin plädierte er für die Wiedereinführung der Konfessionsschulen und gegen die Trennung von Staat und Kirche. Eine enge Zusammenarbeit mit der katholischen Kirche auf politischem und sozialem Gebiet hielt er allerdings nicht für wünschenswert, wobei sein tiefsitzendes Mißtrauen gegen den ,,Ultramontanismus“ deutlich zum Vorschein kam. Interessant ist, daß Niemöller zu diesem Zeitpunkt noch für die Reorganisation der evangelischen Kirche auf der Grundlage der föderalistischen Verfassung des Deutschen Evangelischen Kirchenbundes von 1922 eintrat.

Nach dem Gespräch schrieb Knappen in einem Bericht an Murphy, Niemöller sei ,a sincere and courageous but somewhat provincially minded pastor". Man müsse sehr genau zwischen dem Mann der Kirche, dem Respekt gebühre, und dem politisch ambitionierten Ex-Offizier, den es sorgfältig zu überwachen gelte, unterscheiden. Weiterhin empfahl Knappen, die Ansichten Niemöllers zur Neuordnung der evangelischen Kirche gewissenhaft zu prüfen, alle offiziellen Verhandlungen aber über die Bischöfe Wurm und Meiser abzuwickeln ${ }^{17}$. Einen Tag später ordneten die politischen Berater der amerikanischen und der britischen Militärregierung, Murphy und Ivone Kirkpatrick, die Freilassung Niemöllers an ${ }^{18}$. Wäre ihnen bekannt gewesen, daß Niemöller seit 1924 NSDAP gewählt hatte, wäre die Entscheidung vermutlich anders ausgefallen.

Zweifellos verscherzte sich Niemöller, dessen Naturell kein diplomatisches Taktieren zuließ, mit seinem forschen Auftreten viele Sympathien. Dazu gehörte auch, daß Ende Juli ein von amerikanischen Militärgeistlichen arrangierter Vortrag vor tausend Angehörigen der US-Besatzungsbehörden in Frankfurt, der ein gutes Forum zur Selbstdarstellung bruderrätlicher Reformbestrebungen geboten hätte, im letzten Moment von höchster Stelle verboten wurde ${ }^{19}$. Selbst ein so wohlgesonnener Freund wie George Bell, der Bischof von Chichester, urteilte Ende 1945: ,Wenn ein Held aufhört, ein Held zu sein, wird er zum Problem. Seit Martin Niemöllers Befreiung ist das Niemöller-Problem entstanden. "20 Gerade weil die Militärregierung mit der Zielsetzung der Demokratisierung angetreten war, die man im kirchlichen Raum mit indirekten Mitteln fördern wollte, mußte Niemöller wegen seiner ungeschickten politischen Außerungen auf Ablehnung stoßen, was seinen Gegenspielern, den Führern der , intakten" Landeskirchen, zugute

${ }^{16}$ NA, RG 84, 737/2, OSS-Report No. L-80 vom 20.6.1945. Zur Stellung der evangelischen Kirche bei Beginn des Zweiten Weltkriegs vgl. Brakelmann, Kirche im Krieg. Zum Schweigen der Kirchen anläßlich des sog. Röhm-Putsches vgl. Scholder, Kirchen, Bd.2, S. $221 \mathrm{ff}$.

17 NA, RG 84, 737/2, Report on Interview with Pastor Niemoeller vom 19.6.1945.

${ }^{18} \mathrm{NA}, \mathrm{RG}$ 84, 737/3, Aktennotiz an SHAEF vom 19.6.1945.

19 Vgl. Boyens, Kirchenpolitik, S. 25f., Anm. 69; Knappen, Peace, S. $112 \mathrm{ff}$.

20 Zit. nach Besier, Geschichte, S. 14. 
kam. Ohne es bewußt zu wollen, förderte die Militärregierung damit die mehr restaurativen Kräfte, die ohnehin über die besseren organisatorischen und finanziellen Voraussetzungen verfügten als die Bruderräte. Noch im September 1945 berichtete Lucius Clay, der stellvertretende Militärgouverneur der US-Zone, nach Washington: , While permitting Niemöller to take active leadership in religious affairs, we have not felt it is advisable to utilize his services in other fields as yet. While his anti-Nazi stand was demonstrated fully by his own actions, it is still too early to predict as to his wholehearted rejection of the militaristic and nationalistic concepts of the former German state. “21

Wesentlich einfacher gestaltete sich hingegen für die Militärregierung das Arrangement mit Meiser und Wurm. Sie dachten politisch ebenso konservativ wie Niemöller, hatten aber das Glück, nicht im Rampenlicht der amerikanischen Presse zu stehen. Am 19. Mai hatte Knappen eine erste Unterredung mit Meiser, dem Sprecher des konfessionsbewußten Luthertums und treibenden Mann des Rates der Evangelisch-Lutherischen Kirche Deutschlands (Lutherrat). Er informierte Knappen aus seiner Sicht über die innerkirchliche Lage und stellte insbesondere das Einigungswerk Wurms heraus. Seinerseits erfuhr Meiser, daß die Militärregierung der ,,Fühlungnahme bzw. [dem] Zusammenschluß der deutschen Landeskirchen untereinander nicht hinderlich sein" wolle, ,,doch sei die Zeit dafür noch nicht gekommen“. Meisers kurze Aktennotiz endete mit dem Passus: ,Ich bitte ihn, den General Eisenhower über die allgemeine Lage zu unterrichten, Gefahr der Hungersnot, Verelendung des Volkes, Not der wegen Parteizugehörigkeit Entlassenen, Gefahr der Bolschewisierung. "22 Diese vier Stichworte umreißen schlaglichtartig die unter evangelischen wie katholischen Kirchenführern weitverbreitete politische Grundorientierung, die die Kritik der Entnazifizierungspolitik und die Bekämpfung des Kommunismus bereits zehn Tage nach der bedingungslosen Kapitulation zu den vordringlichsten Anliegen der Kirche zählte. Zwei Monate später sollte Meiser in einer gemeinsamen Eingabe mit Kardinal Faulhaber heftig gegen die amerikanischen Entnazifizierungsmaßnahmen protestieren ${ }^{23}$.

$\mathrm{Zu}$ den manchmal so entscheidenden Zufälligkeiten, die den ersten Besatzungswochen ihr eigenes Gepräge gaben, gehörte es, daß Meiser die Festnummer des Nürnberger Gemeindeblattes anläßlich seiner Amtseinführung als Landesbischof 1933, die ihn mit der bayerischen NS-Prominenz abbildete, einem Religious Affairs Officer zeigte, der den wohlwollenden Rat erteilte, ,,sie den übrigen Stellen nicht vorzulegen“ ${ }^{24}$. Die Episode beleuchtet die politische Naivität Meisers in einer Zeit, als Knappen und Murphy im Anschluß an ihre Unterredungen mit ihm und Faulhaber das Konzentrationslager Dachau aufsuchten und sich über die Ermordung katholischer und evangelischer Pfarrer aus den von Deutschland besetzten Gebieten unterrichten ließen ${ }^{25}$ - eine Geste, zu der sich kein deutscher Kirchenführer bereit fand.

21 NA, RG 260, 5/341-3/37, Clay an General Archer Lerch vom 21.9.1945. Wie tief das Mißtrauen $\mathrm{saß}$, zeigen kritische Presseberichte über Niemöllers große Vortragsreise durch die USA vom Dezember 1946 bis März 1947. Vgl. New York Times vom 10.12.1946, Globe Democrat vom 3. 12. 1946, P.M. vom 21.1. 1947. Weitere Presseausschnitte: WCC, Dr. Martin Niemöller.

22 LKAN, NL Meiser 212, Aktennotiz Meisers vom 19.5.1945. Vgl. Kap. IV/2.

23 Vgl. Kap. III/2.

${ }^{24}$ LKAN, NL Meiser 212, Aktennotiz Meisers über Besprechung mit Captain Landeen vom 15.5.1945.

${ }^{25}$ Vgl. Knappen, Peace, S. 95. 
Am 10. Mai übergab Wurm der Militärregierung in Stuttgart ein Memorandum, in dessen Mittelpunkt unmittelbar kirchliche Interessen standen: die freie Amtsausübung der Pfarrer, die baldige Entlassung aller kriegsgefangenen Pfarrer, Theologiestudenten und kirchlichen Angestellten, die Herausgabe eines Gemeindeblattes und die Rückgabe ehemaliger kirchlicher Einrichtungen. Weitere Punkte betrafen die Wiedereröffnung der kirchlichen Kindergärten, das kirchliche Wohlfahrtswesen und die Fortzahlung der Staatsleistungen, deren monatliche Höhe Wurm mit 290000 RM bezifferte. Daran schlossen sich einige Bitten für die Bevölkerung an. An erster Stelle stand die Bitte, ,,Unschuldige nicht für die Verfehlungen der Partei zu bestrafen", was als vorsichtige Kritik an ersten Entlassungsmaßnahmen verstanden werden kann. Außerdem setzte sich Wurm für die menschenwürdige Behandlung der deutschen Kriegsgefangenen, für die Freilassung Minderjähriger und für eine unabhängige deutsche Rechtspflege ein ${ }^{26}$. Ein paar Tage später wiederholte er in einem Gespräch mit Captain Maley die kirchlichen Anliegen und erreichte die Zusage, daß Briefe an ausländische Kirchenführer mit der militärischen Kurierpost nach Genf und Großbritannien befördert werden konnten. Zugleich äußerte Wurm, er wolle sobald wie möglich Kontakt mit den Kirchenführern Meiser, August Marahrens, Friedrich von Bodelschwingh sowie Hanns Lilje und Eugen Gerstenmaier aufnehmen; keiner von diesen war ein Vertreter des Reichsbruderrats. Nicht minder aufschlußreich für die politische Orientierung war die Erklärung, daß zur Abfassung neuer Lehrpläne und Schulbücher nur ,,gutwillige und erfahrene Glieder aus Kirche und Volk" herangezogen werden sollten, ,,damit nicht die Aspekte der Emigration und die Gesichtspunkte anderer Völker Berücksichtigung finden"27.

Auch in der ersten Unterredung mit Militärgouverneur Dawson am 18. Juni lenkte Wurm nach der Erörterung kirchlicher Anliegen das Gespräch auf politische Themen. Mit besonderem Nachdruck setzte er sich dabei für die Freilassung internierter SS-Männer ein, da sie häufig zwangsrekrutiert worden seien, und wandte sich gegen die generelle Entlassung von NSDAP-Mitgliedern. Als Sohn eines lutherischen Pastors zeigte Dawson ein überraschend großes Verständnis und betonte, daß er ,, befürwortende Eingaben des Herrn Landesbischof für solche Beamte positiv berücksichtigen [werde], wie er überhaupt dankbar sei für entsprechende Vorschläge des Herrn Bischof im Blick auf den Wiederaufbau unserer Verwaltung und unseres Schulwesens". Zugleich ordnete Dawson, wie der Bericht von Oberkirchenrat Pressel (dessen NSDAP-Mitgliedschaft Dawson sicherlich nicht bekannt war), dankbar vermerkte, die Beschlagnahmung zweier Privatfahrzeuge zur ständigen Verfügung des Stuttgarter Oberkirchenrats an ${ }^{28}$; Niemöller war ein Auto verweigert worden ${ }^{29}$. Die Stellung der Fahrzeuge und des nötigen Benzins sowie entsprechender Passierscheine erlaubten Wurm und einigen Begleitern die Informationsreise zur Vorbereitung der Kirchenführerkonferenz von Treysa Ende August 1945.

Am 22. Juni traf Wurm in Frankfurt Knappen, der mit einem Empfehlungsschreiben die Reise überhaupt erst ermöglicht hatte. Während der Unterredung betonte Wurm, die Kirche als Institution strebe die Errichtung einer christlichen Ordnung an, die dem Nationalsozialismus wie dem Kommunismus gleichermaßen feindlich gegenüberstehe. Zur

\footnotetext{
${ }^{26}$ LKAS, NL Hartenstein 49, Wurm an Militärregierung Stuttgart vom 10.5.1945.

${ }^{27}$ LKAS, NL Hartenstein 50/1, Aktennotiz über Unterredung mit Captain Maley am 15. 5. 1945.

${ }^{28}$ LKAS, NL Hartenstein 52, Aktennotiz Pressels über Unterredung mit Dawson am 18.6.1945.

29 NA, RG 84, 737/2, Knappen, Report on the Niemoeller case up to date vom 10.7.1945.
} 
Erfüllung ihrer Aufgaben bedürfe es allerdings folgender Voraussetzungen - Wurm las von einer vorbereiteten Liste ab: Respektierung des Sonntags als Feiertag, christliche Erziehung in den Schulen und christliche Jugendarbeit, Zulassung kirchlicher Sozialarbeit, Wiedereröffnung der theologischen Seminare, Kontakte zu ausländischen Kirchen, Entlassung von Pfarrern und Theologiestudenten aus der Kriegsgefangenschaft, Ausweitung der religiösen Presse und regelmäßige Benzinzuteilungen. Hinsichtlich der Neuordnung der evangelischen Kirche nannte Wurm in Stichworten: Selbstreinigung von Nazi-Elementen, Reorganisation auf zentraler Leitungsebene und Rückkehr zur Kirchenverfassung von 1922. Knappen betonte den guten Willen der Militärregierung, gab aber zu verstehen, daß die Militärregierung weder kirchliche noch laizistische Gruppen einseitig bevorzugen wolle. Die Regelung aller religiösen Fragen, womit er vor allem auf die Problematik der Konfessionsschulen anspielte, solle dem deutschen Volk selbst überlassen werden. In seinem Bericht notierte Knappen anschließend die Befürchtung, Wurm und Niemöller, die sich am gleichen Tag das erste Mal wiederbegegnet waren, könnten sich an die amerikanische Offentlichkeit wenden, um gegen die Besatzungspolitik zu protestieren $^{30}$.

Einen Tag später, am 23. Juni, hatte Wurm eine wichtige Unterredung mit Murphy, an der auch Pressel und Knappen teilnahmen. Wiederum verlas der Bischof, nachdem er seine Dankbarkeit über das Ende der Unterdrückung der Kirche ausgesprochen und gleichzeitig Ausschreitungen französischer Besatzungstruppen scharf verurteilt hatte, eine Bittliste. An erster Stelle stand die Einberufung der geplanten Kirchenführerkonferenz, an fünfter Stelle die Entnazifizierungsfrage. Wurm setzte sich nachdrücklich für die Beibehaltung derjenigen Beamten ein, die nur ,,nominelle Parteimitglieder" gewesen seien. Eine gerechte Beurteilung könnten nur die Ortspfarrer abgeben, nicht aber politisch völlig einseitig zusammengesetzte ,Antifaschistische Ausschüsse": ,He said that sometimes these bodies were composed of people who had been long in concentration camps and did not know community sentiment as did the churchmen who could speak for the majority element in a given community." Diese Ausführungen und ein Gespräch mit Pressel, der den antikommunistischen Charakter der kirchlichen Jugendarbeit besonders hervorhob, veranlaßten Knappen erneut, vor möglichen politischen Umtrieben unter dem Deckmantel der Kirche zu warnen ${ }^{31}$. Wie viele amerikanische Besatzungsoffiziere aus akademischem Milieu hegte Knappen keine Sympathien für den Kommunismus, reagierte aber empfindlich, wenn sich Kirchenvertreter offen als Antikommunisten empfahlen und jenen Bündnispartner der USA kritisierten, der am meisten unter der deutschen Kriegsführung zu leiden gehabt hatte.

Dennoch befürworteten Murphy und Kirkpatrick am 27. Juni in einer gemeinsamen Stellungnahme die Einberufung der von Wurm vorbereiteten Kirchenführerkonferenz: ,Bishop Wurm has a good record of anti-Nazi activity. From the point of view of public opinion in the US and Britain, there is some advantage in using him to pick up the threads of the Protestant church. "32 Damit war die entscheidende Weichenstellung für den Aufstieg Wurms zum Ratsvorsitzenden der neugegründeten Evangelischen Kirche in

30 NA, RG 84, 737/2, Report on Interview with Bishop Wurm of Wuerttemberg vom 23.6.1945.

31 NA, RG 84, 734/23, Report on Conferences with Bishop Wurm's Party vom 26.6. 1945. Vgl. allg. Niethammer/Borsdorf/Brandt, Arbeiterinitiative 1945.

32 NA, RG 84, 737/2. 
Deutschland (EKD) gestellt. Daß die Bekennende Kirche die Führung übernehmen werde, stand ohnehin außer Frage; doch vor die Alternative Wurm oder Niemöller gestellt, favorisierte man aus politischen Gründen Wurm. Dieser besaß nicht nur im deutschen Protestantismus einen starken Rückhalt, sondern auch - was vielleicht noch wichtiger war - in der Okumene. Am 2. Juli telegraphierte Murphy nach Washington und erbat die offizielle Genehmigung, die am 21. Juli erfolgte. Das State Department befürwortete allerdings, wie Murphy selbst, eine demokratisch-synodal legitimierte Wahl der Kirchenvertreter durch die Gemeinden ${ }^{33}$. Die innere Zersplitterung der evangelischen Kirche und die chaotischen Verkehrsverhältnisse arbeiteten jedoch für Wurm, der auf die Neuordnung der evangelischen Kirche ,,von oben“" setzte.

Am 30. Juni und 1. Juli unterrichtete Wurm in Frankfurt die Militärregierung über die Ergebnisse seiner Rundreise. Präses Karl Koch und Bodelschwingh seien mit der Einberufung der Kirchenführerkonferenz für Ende August in Treysa einverstanden und hätten ihn als den Führer des deutschen Protestantismus mit der Vorbereitung beauftragt. Gegenüber den Amerikanern trat Wurm sehr selbstbewußt auf: ,, The Bishop replied that he suspected to be elected head of the Protestant church. Niemöller would be his ,right hand'. ,I am old', said the Bishop, and Niemöller is the logical one to succeed me'."34 Auf Rückfrage erklärte Wurm, daß er die Auswahl der Teilnehmer persönlich treffen werde und daß einige Kirchenführer, die den Deutschen Christen angehört oder mit dem NSRegime eng zusammengearbeitet hätten, keine Einladung erhalten sollten; dies gelte unter Umständen auch für Bischof Marahrens aus Hannover. Insgesamt rechnete Wurm mit 30 bis 40 Personen, die sich aus den amtierenden Kirchenführern der Westzonen und ihren Begleitern sowie aus Bodelschwingh und Niemöller zusammensetzen sollten. Tatsächlich erschienen jedoch, mit oder ohne Einladung, über 100, unter ihnen auch Marahrens.

In Frankfurt benutzte Wurm erneut die Gelegenheit, sich über das Verhalten der französischen Truppen zu beschweren, und kritisierte die Internierung von SS-Männern und die Entlassung nationalsozialistischer Beamter. Insbesondere setzte er sich für hohe Beamte und Industrielle ein, die die NSDAP häufig zum Parteieintritt gezwungen habe: ,I feel that many of these former ,Nazis' are indisponsible, because they cannot be replaced. You are denazifing too quickly and putting people into positions who can't know their jobs. “" 35 Die gleichen Punkte sprach Wurm am nächsten Tag nochmals an. Dabei setzte er sich unter anderem für den entlassenen Stuttgarter Bürgermeister Karl Strölin ein, der 1933 von den Nationalsozialisten eingesetzt worden war und das Deutsche Auslandsinstitut geleitet hatte ${ }^{36}$. Unermüdlich trug Wurm sein politisches Credo vor, wonach Nutznießer der Not des Volkes und der Entnazifizierung nur unliebsame, radikale Elemente sein könnten; gemeint waren damit wohl die Kommunisten.

${ }^{33}$ Vgl. Boyens, Kirchenpolitik, S. $36 \mathrm{f}$.

${ }^{34}$ NA, RG 84, 737/1, Lapp, Report on Conference with Bishop Wurm's Party vom 30. 6. 1945. Zur Vorbereitung der Konferenz vgl. Smith-von Osten, Treysa, S.37ff., Thierfelder, Wurm.

35 Ebenda.

${ }^{36}$ Anscheinend besaß der neue Stuttgarter Oberbürgermeister Arnulf Klett nicht das Vertrauen Wurms; wobei möglicherweise konfessionelle Rivalitäten mitspielten, da Klett vom Rottenburger Bischof Sproll unterstützt wurde. Vgl. Sproll an OMGWB vom 25.7.1945, in: Akten deutscher Bischöfe, Bd.6, S.607f. 
Für Jacob D. Beam, einen engen Mitarbeiter Murphys, stand nach den Gesprächen fest: ,,There is no doubt that Bishop Wurm is nationalistic in his outlook and will try all along the line to get an amelioration of the restrictions and terms imposed upon his people. " $37 \mathrm{Zu}$ einer differenzierteren Einschätzung war Murphy selbst nach getrennten Unterredungen mit Wurm und Niemöller gelangt. In einem Bericht an den amerikanischen Außenminister vom 25. Juni wies er vor allem auf das starke Selbstbewußtsein der Kirchenführer hin: , Both are, I believe, ardent German patriots who have violently disagreed with policies of the National Socialist Party in the past but who are also capable of similar violent disagreement with the Allied occupation authorithies. [...] Niemoeller is far more aggressive than Bishop Wurm. Both, I believe, can be useful to our Military Government authorities, but there is doubt [...] that the relations with them in the future will be entirely smooth and easy. "38 Diese Vorhersage sollte sich als zutreffend erweisen. Bereits während der ersten Begegnungen zeichnete sich der Konfliktpunkt Entnazifizierung ab, der das Verhältnis bald schwer belasten sollte.

Otto Dibelius, der neue Bischof von Berlin-Brandenburg, gab während einer Unterredung mit Knappen am 28. Juli, in deren Mittelpunkt ebenfalls die geplante Konferenz in Treysa stand, deutlich zu verstehen, daß er von einer synodalen Legitimierung der angestrebten Neuordnung nichts halte. In Übereinstimmung mit Niemöller und Wurm, aber in starkem Kontrast zu Meiser, betonte Dibelius allerdings seinen Willen zur Selbstreinigung der Kirche von nationalsozialistischen und deutschchristlichen Einflüssen. Mehr am Rande kamen das Flüchtlingselend, die Frage der Konfessionsschulen und die Druckerlaubnis für kirchlich-religiöse Schriften zur Sprache. Ein Punkt, der Knappen aufhorchen ließ, waren die politischen Ausführungen des Bruderratsmitglieds. Dibelius versicherte, daß der Kommunismus nach den Gewalttaten der russischen Truppen in Deutschland keine Chance mehr habe. Hier sah Dibelius wesentlich klarer als seine Kollegen aus den Westzonen, die vielfach den Untergang des ,,christlichen Abendlandes“ befürchteten. Andererseits betonte aber auch er: ,Democracy will not take root in Germany because: 1 . it is a foreign ideology. 2 . because of Germany's experience with the weak Weimar Republic, democracy is associated in the German mind with unemployement and ineffective foreign policy. "Damit brachte Dibelius vorsichtig, aber doch unüberhörbar die antidemokratischen Vorbehalte weiter Kreise, nicht zuletzt seine eigenen, zum Ausdruck. Dieselbe politische Beurteilung trug der Berliner Bischof wenig später Stewart Herman vor, der als Abgesandter des Okumenischen Rates der Kirchen im Spätsommer 1945 mehrfach die US-Zone bereiste. In dieser Situation sah Dibelius, der 1926 die bekannte Programmschrift „Das Jahrhundert der Kirche“ verfaßt hatte, die große Chance der Kirche und ihres Rechristianisierungsprogramms: , , Therefore in order to prevent the revival of Nazism, the Occupying Powers should assist the church which offers its supporters traditional ideology rooted in Germany which will fill the vacuum left by the collapse of the Hitler movement. "39

${ }^{37}$ NA, RG 84, 737/1, Memorandum of a Conversation with Bishop Wurm of Wuerttemberg vom 1.7.1945. Murphy war bei dieser Begegnung - entgegen Boyens, Kirchenpolitik, S.36 - nicht anwesend.

38 NA, RG 84, 737/4, Interviews with Pastor Niemöller and Bishop Wurm vom 25.6.1945.

${ }^{39}$ NA, RG 84, 737/1, Report on Conference with Dr. Dibelius vom 28.7.1945. Vgl. WCC, Germany 284(43), Bericht Hermans über Unterredung mit Dibelius vom 9. 8. 1945. Die zitierten Berichte amerikanischer Besatzungsoffiziere über erste Begegnungen mit evangelischen Kirchen- 
Welchen Eindruck die ersten persönlichen Begegnungen mit Kirchenführern aus der Bekennenden Kirche bei den zuständigen amerikanischen Offizieren hinterließen, läßt sich dem rückblickenden Urteil Knappens aus dem Jahre 1946 über seine Unterredung mit Dibelius entnehmen: ,,Conversations such at this did little to weaken the feeling that the element in control of the reorganized German Evangelical Church, while demonstrably anti-Nazi, was nationalistic and Junker-monarchical rather than international and liberal-democratic in its political outlook. " 40 Besonders die sofort einsetzende Fürsprache für entlassene Nationalsozialisten mußte in der Militärregierung ernste $Z$ weifel an der Demokratiebereitschaft der evangelischen, aber auch der katholischen Kirche hervorrufen. Der katholischen Kirche bescheinigte Knappen allerdings ein wesentlich diplomatischeres Auftreten. Hinzu kam, daß 1945/46 viele Besatzungsoffiziere noch an den Erfolg der Potsdamer Beschlüsse hinsichtlich der Viermächtekontrolle Deutschlands glaubten und die ständigen Warnungen der Kirchenführer vor dem Kommunismus als unzulässige Kritik am sowjetischen Kriegsverbündeten empfanden.

Die Gesprächsniederschriften lassen die Vorbehalte prominenter evangelischer Kirchenführer gegenüber einer weltanschaulich pluralistischen und demokratisch verfaßten Gesellschaft deutlich erkennen. Der Begriff Demokratie wurde in den Unterredungen sorgfältig vermieden oder ausschließlich negativ gebraucht. Die Frage, welchen Beitrag die Kirche zur Bewältigung der Probleme der demokratischen Neuordnung leisten könne, hat sich den Kirchenführern kaum gestellt. Ihr Hauptinteresse galt der Durchsetzung unmittelbar kirchlicher Anliegen; wenn sie jedoch die Gelegenheit zu politischer Stellungnahme benutzten, dann um die Entnazifizierungspolitik zu kritisieren oder vor linken Kräften und Strömungen zu warnen. Konkrete Neuordnungskonzeptionen, die über den allgemeinen Wunsch, der Kirche wieder einen herausragenden gesellschaftlichen Einfluß zu sichern, hinausgingen, lassen sich hingegen nicht erkennen. Bereits 1941 hatte Dietrich Bonhoeffer, erschreckt über den Mangel an Zukunftsperspektiven für die Zeit nach Hitler, als Erklärung festgehalten: , Die absolute Ungesichertheit der menschlichen Existenz führt [...] bei den Christen fast überall zum völligen Verzicht auf jeden Gedanken an die Zukunft, was wiederum eine stark apokalyptische Haltung zur Folge hat. Unter dem Eindruck der Nähe des Jüngsten Tages geht der Blick für die geschichtliche Zukunft leicht verloren." 4 1

Aus oppositionellen kirchlich-protestantischen Kreisen liegt zur Frage der Zukunft nur die „Freiburger Denkschrift" von 1943 vor. Sie war auf Anregung Bonhoeffers von einer Freiburger Professorengruppe unter Mitarbeit von Dibelius und dem Theologen Helmut Thielicke verfaßt worden. $\mathrm{Zu}$ den beteiligten Professoren gehörten der Historiker Gerhard Ritter und die Nationalökonomen Constantin von Dietze, Walter Eucken und Adolf Lampe; die Denkschrift spiegelte das hierarchisch-autoritär geprägte Staatsund Gesellschaftsbild der Verschwörer des 20. Juli 194442. Doch 1945, nach der bedin-

führern sind mittlerweile in der vom Verfasser bearbeiteten Edition: Die evangelische Kirche nach dem Zusammenbruch, leicht zugänglich.

${ }^{40}$ Knappen, Peace, S. 101. Vgl. auch S. 145.

41 Zit. nach Bethge, Bonhoeffer, S. 871.

${ }^{42}$ Helmut Thielicke (Hrsg.), In der Stunde Null. Die Denkschrift des Freiburger ,BonhoefferKreises", Tübingen 1979. Vgl. Bethge, Bonhoeffer, S. 871 ff. Vgl. allg. Hans Mommsen, Gesellschaftsbild und Verfassungspläne des deutschen Widerstands, in: Hermann Graml, Widerstand im Dritten Reich. Probleme, Ereignisse und Gestalten, Frankfurt 1984, S.14-91. 
gungslosen Kapitulation, waren die politischen Voraussetzungen für ein eigenständiges Handeln des nationalkonservativen Widerstandes nicht mehr gegeben. Die militärische Niederlage des NS-Regimes traf die Bekennende Kirche nahezu unvorbereitet. Sie wurde zudem mit sehr ambivalenten Gefühlen wahrgenommen, bedeutete sie doch einerseits das Ende der zunehmenden Verdrängung der Kirchen aus dem öffentlichen Leben, andererseits aber auch den völligen Zusammenbruch des deutschen Staates. Letzteres mußte den obrigkeitsstaatlich und national geprägten deutschen Protestantismus schwer erschüttern. Die bedingungslose Kapitulation und der damit verbundene Beginn der Besatzungsherrschaft konnten deshalb nicht rundum als Befreiung empfunden werden. Eher erblickte man darin, wie Meiser in einem Rundschreiben an die bayerische Pfarrerschaft vom 7. Mai 1945 formulierte, die Vollendung der ,,deutschen Tragödie“"433.

${ }^{43}$ LKAN, LKR 274. Vgl. Vollnhals, Landeskirche, S. $143 \mathrm{ff}$. 